\title{
Functional Activity of Circulating Phagocytes as Potential Pretreatment Marker of Tumor Drug Resistance
}

\author{
Iryna V. Prokhorova1 ${ }^{\circledR}$, Olga V. Yurchenko ${ }^{1}$, Olga N. Pyaskovskaya ${ }^{1}$, \\ Igor N. Todor ${ }^{2}$, Galina I. Solyanik ${ }^{1}$
}

${ }^{1}$ Laboratory of Molecular and Cellular Mechanisms of Metastasis, R.E. Kavetsky Institute of Experimental Pathology, Oncology and Radiobiology of NASU, Kyiv, Ukraine

${ }^{2}$ Department of Monitoring of Tumor Process and Therapy Design, R.E. Kavetsky Institute of Experimental Pathology, Oncology and Radiobiology of NASU, Kyiv, Ukraine

Email: oncom@online.ua

How to cite this paper: Prokhorova, I.V., Yurchenko, O.V., Pyaskovskaya, O.N., Todor, I.N. and Solyanik, G.I. (2019) Functional Activity of Circulating Phagocytes as Potential Pretreatment Marker of Tumor Drug Resistance. Journal of Biosciences and Medicines, 7, 1-15.

https://doi.org/10.4236/jbm.2019.77001

Received: June 4, 2019

Accepted: July 5, 2019

Published: July 8, 2019

Copyright $\odot 2019$ by author(s) and Scientific Research Publishing Inc. This work is licensed under the Creative Commons Attribution International License (CC BY 4.0).

http://creativecommons.org/licenses/by/4.0/

\begin{abstract}
The aim of this work was a study of the functional activity of neutrophils and peripheral blood monocytes in rats with the transplanted Walker carcinosarcoma as potential predictors of the sensitivity of this tumor to doxorubicin treatment. This study provides an evidence that such indices of the functional activity of circulating phagocytes of the tumor-bearing rats as the quantity and the phagocytosis intensity of monocytes, as well as the intensity of ROS production by monocytes and neutrophils, may reflect the degree of sensitivity of the tumor to doxorubicin. So it was shown that the growth of the resistant tumor caused a significant increase of the number of circulating phagocytic cells and the intensity of phagocytosis by more than $100 \%(\mathrm{p}<0.001)$ compared with the corresponding indices of intact rats and rats with the parental variant of the tumor. The ability of blood monocytes and neutrophils of rats with a resistant tumor to produce ROS was also significantly different from that in intact rats and animals with the parental carcinosarcoma variant. The predictive value of these indices is especially important in the dynamic monitoring of the development of tumor drug resistance during long-term cancer chemotherapy. Considering the standard $2-3$ week interval between the courses of cancer therapy and the short lifetime of circulating phagocytes, an assessment of the indices of their functional activity before each subsequent course can be considered as a pretreatment assessment. Meanwhile, further studies are needed to determine the spectrum of malignant neoplasms for which the degree of tumor drug resistance correlates with the functional activity of circulating phagocytes.
\end{abstract}




\section{Keywords}

Circulating Phagocytes, Phagocytic Activity, ROS Production, Tumor Drug Resistance

\section{Introduction}

Breast cancer (BC) is one of the most common forms of malignant neoplasms, ranking first among women in cancer-related mortality worldwide [1]. Despite the achievements in early diagnosis and the understanding of the molecular basis of the $\mathrm{BC}$ biology, progression of the disease is observed in almost $30 \%$ of patients with early stages of the disease [2].

Modern tactics for the treatment of cancer patients, including those with BC, are based on three main approaches: surgical, radiation and medicamentous therapy. Depending on the localization of the process, its prevalence, the histological type, and the clinical picture of the disease course, the type of treatment and its goals differ. This is especially true for medicamentous therapy, which is characterized by a large variety and includes traditional cytotoxic therapy, immunotherapy, hormone therapy, and biotherapy. It is the success of medicamentous therapy for the last 20 years that significantly improved the prognosis for BC patients [2].

In general, antitumor agents used in the treatment of patients with $\mathrm{BC}$ are effective at the beginning of therapy in about $80 \%$ of cases of early-stage primary $\mathrm{BC}$ and $50 \%$ of cases of metastatic BC [3]. However, after some time there is observed the cancer progression. At this stage of the disease, the resistance to chemotherapy is expected and registered in almost all cases. Also, in addition to resistance to traditional chemotherapeutic agents, such as cytotoxic ones, cancer cell resistance to targeted preparations is also observed [4].

Presently, acquired resistance is diagnosed during the long course of drug administration. In clinical practice, the main feature of the formation of the drug resistance of malignant tumors is the reduction of the treatment effectiveness.

It is known that the main index of the treatment effectiveness is the survival rate of patients with malignant neoplasms. However, for the evaluation of the direct response of the tumor process to therapy, the criterion of the effectiveness of treatment for solid tumors (Response Evaluation Criteria in Solid Tumors; RECIST) includes the determination of the minimum size of the measured lesions and the maximum number of lesions to be assessed in total and in each particular organ [5]. For this purpose, there is used either computed tomography (CT), or magnetic resonance imaging (MRI), or positron-emission tomography (PET), or plain radiography. Each of these methods has its drawbacks and limitations. For example, PET and radiography, as a rule, are used to confirm the presence, increase or decrease in the number of tumor foci, but not to measure their size. Small tumor lesions (less than $10-15 \mathrm{~mm}$ in size) cannot be measured 
by CT and MRI.

In assessing the effectiveness of therapy, there are also used oncomarkers, whose list grows with each passing year. The most commonly used markers are CA 15-3 (breast cancer), PSA - prostate specific antigen (prostate cancer), CA-125 (ovarian cancer), CEA-cancer embryonal antigen (rectal cancer), AFP-alpha-fetoprotein (hepatocellular carcinoma marker) [6].

Without addressing the problem of the oncological specificity of these markers, it should be noted that their use is ambiguous for assessing the degree of sensitivity or resistance of the tumor to the particular therapy. Thus, a decrease in the level of oncomarkers in the blood of a cancer patient after cancer therapy is usually considered as evidence on its high efficacy and, accordingly, a low degree of resistance of the tumor process. Meanwhile, the high effectiveness of antitumor therapy can also lead to a significant increase of the tumor marker content in the blood due to the destruction of the tumor lesion and the following release of a significant amount of metabolic protein products of tumor cells including tumor markers.

It is supposed that peripheral blood is not just one of the most important carriers of information about the physiological and pathological processes occurring in a body tissue structures, but is also the most accessible system for analyzing this information. Among the many soluble and insoluble elements of the blood, an important place is occupied by the formed elements: leukocytes, erythrocytes, and platelets. It is known that leukocytes (lymphocytes, monocytes, and neutrophils) play a major role in the specific and nonspecific defense of the body against internal and external pathogenic agents, and also make a significant contribution to many pathological processes, including malignant ones. Many studies have shown the existence of an associative relation between certain peripheral inflammatory markers (such as the neutrophil to lymphocyte ratio (NLR) and platelet to lymphocyte ratio) and the prognosis of BC. Based on the analysis of overall survival (OS) and progression-free survival of cancer patients, some authors conclude that there is a relation between the NLR and the resistance of malignant tumors to the action of anticancer drugs [7] [8] [9]. However, it is clear that such a statement requires a more specific evidence base.

Meanwhile, an objective assessment of the effectiveness of treatment of cancer patients and dynamic monitoring of the process of the formation of resistance of malignant tumors to the action of anticancer drugs is extremely important, as it allows to change or stop chemotherapy on time if it is ineffective.

That is why there is an urgent need to develop new, easy-to-implement and clinically significant methods for assessing the degree of susceptibility/resistance of malignant tumors to the action of anticancer drugs.

The aim of this work was an experimental study of the functional activity of neutrophils and peripheral blood monocytes in rats with the transplanted Walker carcinosarcoma as potential predictors of the sensitivity of this tumor to doxorubicin treatment. 


\section{Materials and Methods}

Experimental animals and tumors. The research was conducted on female Wistar rats 2.5 months old weighing of $185.0 \pm 13.8 \mathrm{~g}$, bred in an animal facility at R.E. Kavetsky Institute of Experimental Pathology, Oncology and Radiobiology of the National Academy of Sciences of Ukraine. Animals were kept in vivarium with the standard light and food regimen (water and nutrition ad libitum). In total, 70 animals were used (including rats that were used to development of a doxorubicin-resistant variant of the Walker 256 carcinosarcoma strain).

The research was carried out per under the "Procedure for Conducting Scientific Experiments on Animals by the Scientific Institutions" (Ukraine, 2012), which is per under the provisions of the European Convention on the Protection of Vertebrate Animals Used for Experiments and Other Scientific Purposes (Starburg, 1986).

In the study, two variants of Walker 256 carcinosarcoma were used the initial parental strain and its variant resistant to doxorubicin. The development of doxorubicin-resistant variant of the strain (DOX, Doxorubicin Teva, Pharmachemie BV, the Netherlands) was performed according to the procedure described in [10]. Briefly, at the first step, a $20 \%$ suspension of cells of the original variant of Walker carcinosarcoma was transplanted in the thigh of animals. When the tumor developed to a volume of $0.5 \mathrm{~cm}^{3}$, rats were administered with DOX once daily for 6 days at a total dose of $9.0 \mathrm{mg} / \mathrm{kg}$ [10]. The next day after treatment termination, anesthetized animals were slaughtered under anesthesia (Sedazin, Biowet Pulawy, Poland), the tumors were withdrawn and used to prepare a $20 \%$ tumor cell suspension, which was transplanted to rats for the next stage of resistance formation according to a scheme similar to the first step. In total, 7 stages of tumor transplantation have been performed.

Assessment of tumor sensitivity to doxorubicin. To assess the sensitivity of each variant of Walker carcinosarcoma to the action of DOX, we have used a tumor growth inhibition coefficient $(\mathrm{D})$, which was calculated by the formula: $\mathrm{D}$ $=(\mathrm{Vc}-\mathrm{V}) / \mathrm{Vc} \times 100 \%$, where $\mathrm{V}$ is the average volume of tumor in a group of animals after 6 consecutive daily administrations of DOX at a total dose of 9 $\mathrm{mg} / \mathrm{kg}$, and $\mathrm{Vc}$ is the mean tumor volume in a group of animals not administered with DOX. The volume of tumors in each group was determined on the 9th day of tumor growth (the day after the end of DOX therapy) by the formula $\mathrm{V}=\mathrm{a} \times \mathrm{b} \times \mathrm{c}$, where $\mathrm{a}, \mathrm{b}, \mathrm{c}$ are the maximum diameters of the tumor node in three mutually perpendicular planes.

Structure of research. The cell suspension of the original and resistant variant of Walker carcinosarcoma was transplanted into the thigh of the rats (5 rats for each variant of the tumor). At the $9^{\text {th }}$ day of tumor growth, the animals were decapitated under anesthesia. The tumors were withdrawn for further histological examination. Blood was collected for the determination of leukogram, phagocytic activity and production of reactive oxygen species (ROS) by blood neutrophils and monocytes. 
The indices of leukogram were determined using an automatic hematologic analyzer Partical Counter PCE-210 (ERMA Inc., Japan).

To determine the spontaneous level of phagocytic activity of neutrophils and monocytes, $90 \mu \mathrm{l}$ of blood was collected in a cytometric tube, $20 \mu \mathrm{l}$ of suspension of inactivated and FITC-labeled staphylococcus $(200 \mathrm{mln} / \mathrm{ml})$ were added and the specimens were incubated for 30 minutes in an incubator at $37^{\circ} \mathrm{C}$. After incubation, the samples were triply washed with phosphate buffer and fixed in $0.4 \%$ formalin solution for further cytofluorometric analysis. During the cytometry of each sample, at least 3 thousand cells in three replicates were analyzed. The relative number of phagocytosis-active neutrophils and monocytes and the rate of engulfed bacteria were determined [11].

To determine the level of ROS production by neutrophils and monocytes, $90 \mu \mathrm{l}$ of blood was placed in a cytofluorometer tube, $10 \mu \mathrm{l}$ of 2',7'-dichlorodihydrofluorescein diacetate (DHF-DA, Sigma-Aldrich, USA) was added and the specimens were incubated for 30 minutes in an incubator at $37^{\circ} \mathrm{C}$. After incubation, the samples were triply washed with phosphate buffer and fixed in $0.4 \%$ formalin solution for further cytofluorometric analysis. During the cytometry of each sample, at least 3 thousand cells in three replicates were analyzed. The relative number of neutrophils and monocytes producing ROS and the intensity of ROS production were determined [11].

Histological study of Walker carcinosarcoma. The tumor biopsies of the initial and resistant variants of Walker carcinosarcoma were fixed in $10 \%$ neutral buffered formalin solution for 24 hours, dehydrated in alcohols of ascending concentrations, and embedded into paraffin at a temperature of $56^{\circ} \mathrm{C}$.; then 5 $\mu \mathrm{m}$ thick histological sections were stained with hematoxylin and eosin [12]. Histological examination of the tumor structure and subsequent photography of the preparations were performed using a microscope Axiostar Plus (Zeiss, Germany).

Statistical analysis. Statistical analysis of the data was performed using descriptive statistics, Students t-criterion, Mann-Whitney U-criterion using Microsoft Excel, Statistic and Microcal Origin software. All data are expressed as the mean \pm standard error.

\section{Results}

Characteristics of two variants of Walker's carcinosarcoma, the original and DOX-resistant variants. Transplantation of the parental and DOX-resistant variants of Walker carcinosarcoma resulted in the development of tumors in 100\% of cases. The tumors of both variants were characterized by infiltrative and rapid growth.

One of the key differences between these tumor variants was their sensitivity to the inhibitory effect of DOX. After 6 consecutive daily administrations of DOX, the tumor volume of the parental variant was significantly reduced by $61 \%(\mathrm{p}<0.05)$, while the tumor volume of the resistant variant showed only a tendency to decrease (Figure 1). 


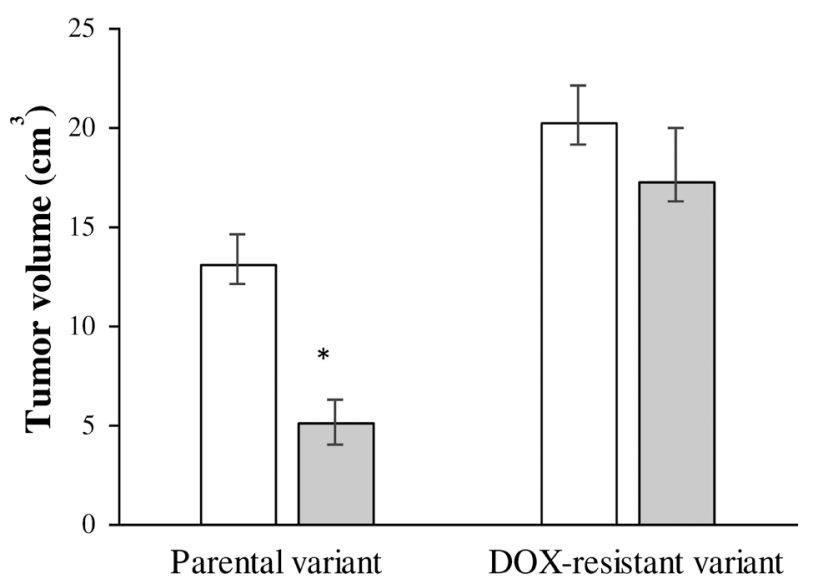

Figure 1. Tumor volume of parental and resistant variants of Walker carcinosarcoma White columns-without DOX treatment; grey columns-after 6 consecutive daily administrations of DOX.

There were also detected some differences in the histological structure of the parental and DOX variants of Walker carcinosarcoma. Histologic studies have shown that tumors, both the parental variant of Walker carcinosarcoma and the DOX-resistant variant, have a heterogeneous structure and consist of an epithelioid component (with small "dark" cells) and a sarcomatous component (with light cells of a large size). Meanwhile, the DOX-resistant tumors (but not the parental variant) are characterized by a higher content of connective tissue component, containing a lesser amount of tumor cells (Figure 2(a), Figure $2(c)$ ). In this layer, there are a large number of granulocytes and macrophages, cell ghosts, and residual cell material. The number of cells in the state of apoptosis was increased. The layers of the epithelioid structures in the resistant variant are determined in a smaller quantity compared with the parental variant (Figure 2(b), Figure 2(d)). In this case, the epithelioid part is represented by cells of a larger size (than in the original version), which form a structured tissue with a large number of newly formed blood vessels.

Blood parameters of rats with parental and DOX-resistant variants of Walker 256 carcinosarcoma. The analysis of the results of the study showed that in the absence of statistically significant differences in the leukocyte counts in animals with tumors, compared with the corresponding indices of intact rats (Table 1), significant changes in the leukocyte formula with a characteristic increase of the percentage of granulocytes and a decrease of the percentage of lymphocytes were recorded. In particular, in rats with the parental tumor variant, the granulocyte counts increased by $64 \%(\mathrm{p}<0.05)$ compared with those of intact animals. The development of the DOX-resistant tumors resulted in a statistically significant increase of the granulocyte counts in rat blood by $136 \%$ compared to intact animals and by $43.9 \%$ compared to this index in the rats with transplanted parental (DOX-sensitive) tumors.

We have shown that the growth of Walker carcinosarcoma was associated with an increase in the NLR in peripheral blood of animals: this index significantly 

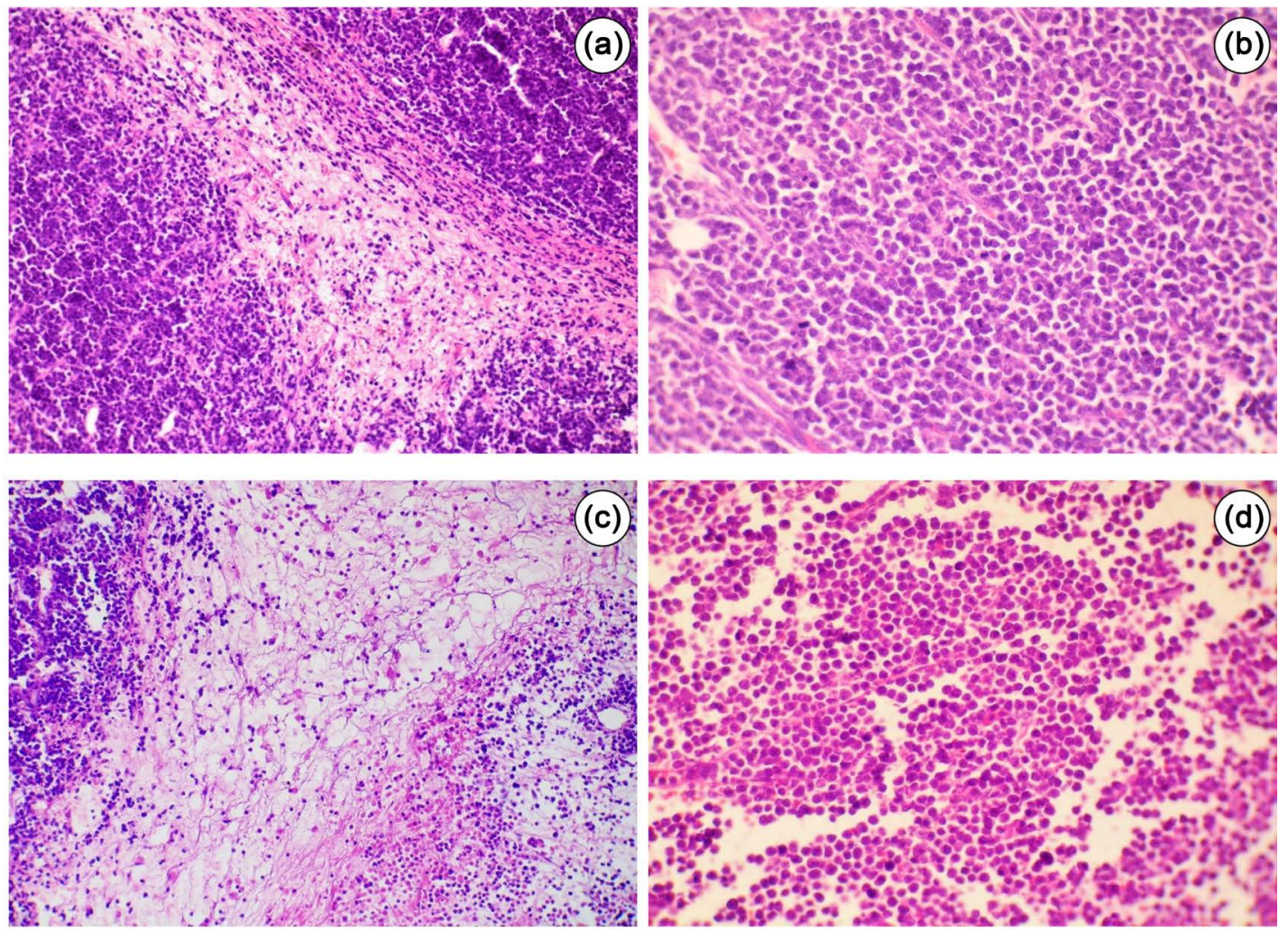

Figure 2. Tumor histological section of parental and DOX-resistant variants of Walker carcinosarcoma; sarcomatous (a) and epithelioid (b) components of parental tumor; sarcomatous (c) and epithelioid (d) components of DOX-resistant variant of tumor. Hematoxylin and eosin stain, $\times 200$.

Table 1. Leukogram indices of intact rats and rats bearing different variants of Walker carcinosarcoma.

\begin{tabular}{cccc}
\hline & & \multicolumn{2}{c}{ Rats with Walker 256 carcinosarcoma } \\
\cline { 3 - 4 } Blood indices & Intact rats & $\begin{array}{c}\text { Parental } \\
\text { (DOX-sensitive) variant }\end{array}$ & $\begin{array}{c}\text { Parental } \\
\text { (DOX-sensitive) variant }\end{array}$ \\
\hline WBC, $\times 10^{3} / \mu \mathrm{l}$ & $10.2 \pm 1.8$ & $9.8 \pm 1.0$ & $13.0 \pm 1.4$ \\
$\mathrm{Ly}, \times 10^{3} / \mu \mathrm{l}$ & $6.7 \pm 1.3$ & $4.9 \pm 0.6$ & $6.1 \pm 0.9$ \\
$\mathrm{Mo}, \times 10^{3} / \mu \mathrm{l}$ & $1.0 \pm 0.2$ & $0.8 \pm 0.2$ & $1.0 \pm 0.2$ \\
$\left.\mathrm{Gr}, \times 10^{3} / \mu \mathrm{l}\right)$ & $2.5 \pm 0.7$ & $4.1 \pm 0.4^{*}$ & $5.9 \pm 0.4^{* \#}$ \\
$\mathrm{Ly}, \%$ & $65.6 \pm 4.6$ & $49.9 \pm 3.0$ & $46.0 \pm 2.1$ \\
$\mathrm{Mo}, \%$ & $9.2 \pm 1.0$ & $7.6 \pm 0.9$ & $8.0 \pm 1.2$ \\
$\mathrm{Gr}, \%$ & $25.2 \pm 5.4$ & $42.5 \pm 3.3$ & $46.0 \pm 1.5$ \\
\hline
\end{tabular}

${ }^{*} p<0.05$ vs. corresponding value of intact rats; ${ }^{*} p<0.05$ vs. corresponding value of rats bearing sensitive variant of tumor.

increased by $122.2 \%(\mathrm{p}<0.05)$, and by $167.2 \%$ in animals with parental or DOX-resistant variants respectively, compared with intact control (Table 2). At the same time, there was no significant difference in the NLR values between the groups of animals with two tumor variants. 
The effect of development of Walker carcinosarcoma variants with different sensitivity to doxorubicin on the indices of functional activity of neutrophils and monocytes. The results of the study showed that the growth of the parental and resistant variants of Walker carcinosarcoma caused significant changes in the functional activity of blood neutrophils and monocytes.

The development of the parental tumor variant led to a significant increase in the percentage of phagocytic monocytes by $107.5 \%(\mathrm{p}<0.01)$ compared with intact animals (Figure 3(a)). In this case, the absolute count of phagocytic monocytes and the intensity of phagocytosis did not differ from the corresponding indices of intact rats (Figure 3(b), Figure 3(c)). At the same time, the growth of the resistant tumor caused a significant increase of the percentage of phagocytic monocytes (by $445 \%$ and $162.7 \%$ ), the absolute count (by $445 \%$ and $162.7 \%$ ) and the intensity of phagocytosis (by 16.6 and 14.4 times) compared with the corresponding indices of intact rats and rats with the parental variant of the tumor (Figures 3(a)-(c)).

Table 2. Neutrophil/lymphocyte ratio in peripheral blood of Wistar rats bearing sensitive and resistant to doxorubicin tumors.

\begin{tabular}{cccc}
\hline Rats & Tumor sensitivity & $\mathrm{M}$ & $\mathrm{m}$ \\
\hline Without tumor & & 0.396 & 0.104 \\
\multirow{2}{*}{ Tumor bearing } & Sensitive & $0.880^{*}$ & 0.13 \\
& Resistant & $1.058^{*}$ & 0.1 \\
\hline
\end{tabular}

${ }^{*} p<0.05$ vs. corresponding value of intact rats.

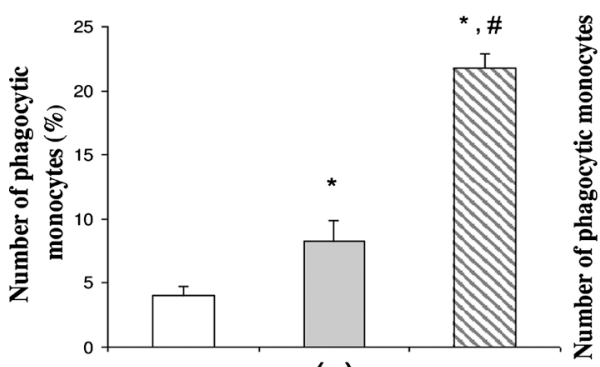

(a)

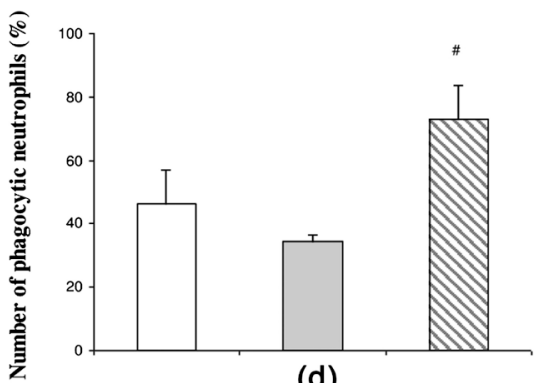

(d)

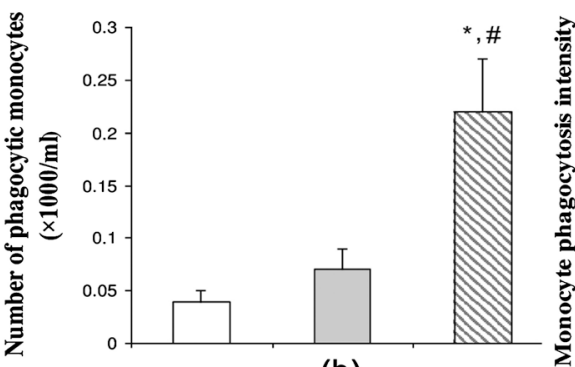

(b)

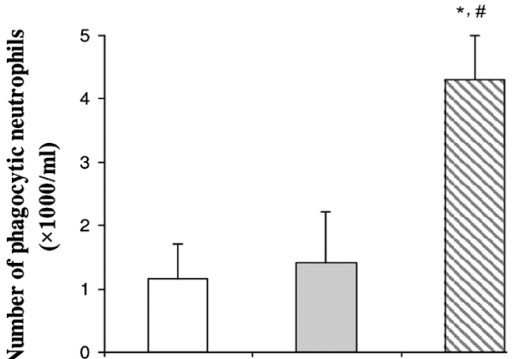

(e)

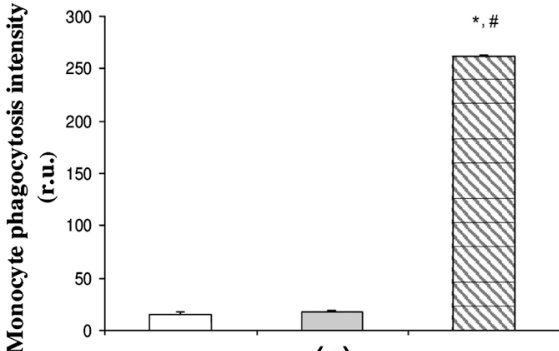

(c)

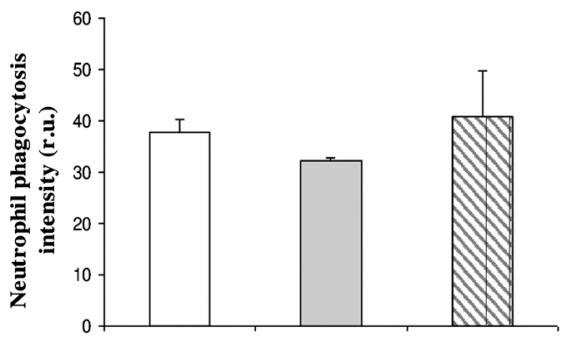

(f)

${ }^{\star} p<0.05$ vs. corresponding value of intact rats; ${ }^{\sharp} p<0.05$ vs. corresponding value of rats bearing sensitive variant of carcinosarcoma.

Figure 3. Indices of phagocytic activity of monocytes and neutrophiles in peripheral blood of intact rats (white column), rats bearing sensitive variant of the tumor (grey column) and rats bearing resistant tumors (columns with diagonal hatch): percentage of monocytes (a) and neutrophils (d); absolute counts of monocytes (b) and neutrophils (e); intensity of monocyte (c) and neutrophils (f) phagocytosis. 
Despite the significant increase of the neutrophil count in the blood of rats with the parental variant of Walker carcinosarcoma, the percentage of phagocytic neutrophils, their absolute counts and intensity of phagocytosis did not differ from the corresponding indices of intact animals (Figures 3(d)-(f)). On the contrary, the development of the DOX-resistant tumor was accompanied by an increase of the percentage of phagocytic cells by $56.6 \%(\mathrm{p}<0.05)$ and by $111.0 \%(\mathrm{p}<0.01)$ compared with the corresponding indices of intact rats and rats with the parental tumor, respectively (Figure 3(d)). Against the background of significant granulocytosis in animals with the DOX-resistant tumor, an increase of the percentage of phagocytic neutrophils caused a significant increase of an absolute count of phagocytic neutrophils more than 3-fold (Figure 3(e)). At the same time, these cells did not differ significantly by the intensity of phagocytosis (Figure $3(\mathrm{f})$ ).

The study has shown that the ability of blood monocytes and neutrophils of animals with a resistant tumor variant to produce ROS was significantly different from that in intact rats and animals with the parental carcinosarcoma variant.

It should be noted that all neutrophils in the blood of intact rats, rats with the parental and resistant variants of the tumor produced ROS (Figure 4(a)). The increase of the absolute count of ROS generating neutrophils and the intensity of ROS production in animals with the parental tumor variant was proportional to the increased neutrophil counts (Figure $4(\mathrm{~b})$ ). The intensity of ROS production by neutrophils in rats with a resistant carcinosarcoma variant was by $642.4 \%(\mathrm{p}$ $<0.001)$ higher compared to the index of intact control, and by $344 \%(\mathrm{p}<0.001)$

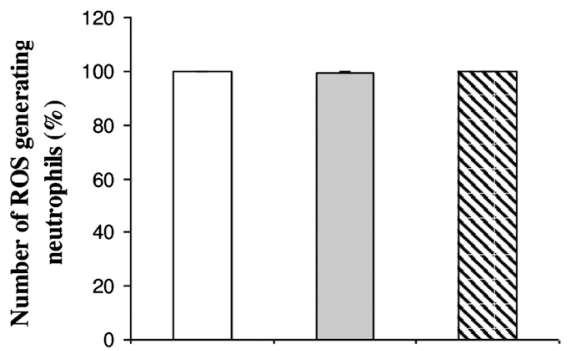

(a)

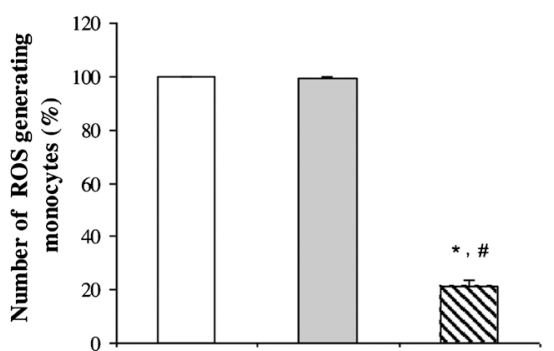

(d)

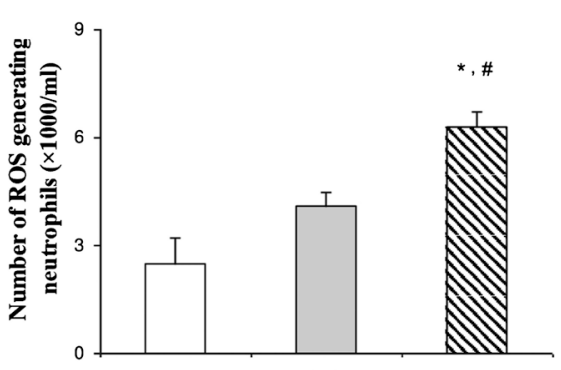

(b)

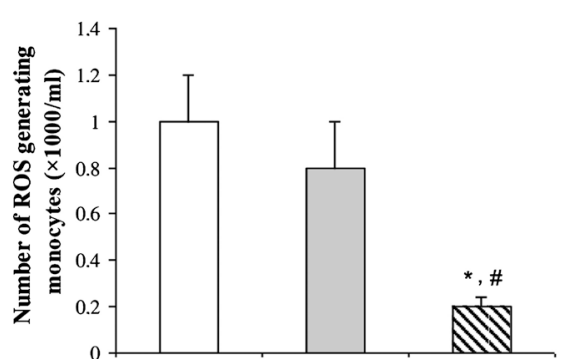

(e)

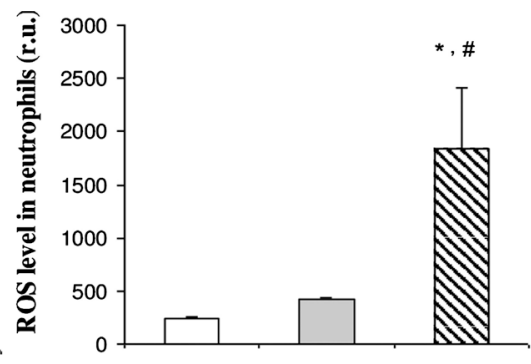

(c)

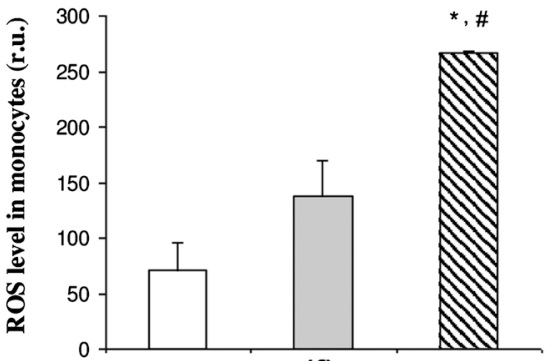

(f)

${ }^{*} p<0.05$ vs. corresponding value of intact rats; ${ }^{*} p<0.05$ vs. corresponding value of rats bearing sensitive variant of carcinosarcoma.

Figure 4. Indices of ROS generating cells in peripheral blood of intact rats (white column), rats bearing sensitive variant of tumor (grey column) and rats bearing resistant tumors (columns with diagonal hatch): percentage of neutrophils (a) and monocytes (d); absolute count (b) of ROS generating neutrophils (b) and monocytes (e), as well as the intensity of ROS production by neutrophils (c) and monocytes (f). 
higher compared to the index in rats with the parental variant of the tumor (Figure 4(c)).

The effect of the growth of the DOX-resistant tumor on the ability of blood monocytes to generate ROS differed from the revealed patterns of neutrophils. First of all, we have observed a significant reduction (more than 75\%) of the counts of ROS-producing monocytes in the blood of rats with the DOX-resistant tumor compared with the corresponding indices of intact animals and rats with the parental tumor variant (Figure 4(d)). Despite this decrease of the ROS-generating monocyte counts, the development of the DOX-resistant Walker carcinosarcoma was associated with an increased intensity of ROS production in these cells by $86 \%,(p<0.05)$ compared to the index in rats with the parental tumor variant and by $263.8 \%(\mathrm{p}<0.001)$ compared with intact control (Figure $4(\mathrm{e})$ ). It should be noted that the number of ROS-generating monocytes and the intensity of ROS production in rats with the parental variant of Walker carcinosarcoma did not differ significantly from the corresponding indices of intact animals (Figure 4(f)).

\section{Discussion}

It is known that the tumor during its growth affects normal organs, tissues and regulatory systems of the body, imposing the peculiarities of its metabolism. That is why the most promising is the development of methods of personalization of medical therapy using extratumoral markers and systems. Blood and its components, which carry information about the general physiological and pathophysiological state of the body, can be considered as a convenient test system for the study of many tumor-related variables. This is especially true for blood cells such as monocytes and neutrophils whose functional status quickly responds to endogenous and exogenous factors, which include humoral factors produced by malignant tumors.

High phenotypic plasticity of neutrophils was noted by many authors [13] [14]. Numerous studies have shown that tumor development can significantly change both the number of circulating and tumor-associated neutrophils and the nature of their effect on the growth and invasiveness of cancer cells [15] [16] [17]. Along with this, a large quantity of soluble factors produced by tumor affects granulopoiesis and causes an appearance of the subset of neutrophils with different migration capacity, and various anti- or pro-tumor properties [18].

In our study, there was also observed a significant increase of the absolute neutrophil counts in the peripheral blood of animals with tumors, more pronounced in a case of a DOX-resistant variant. In general, the growth of both Walker carcinosarcoma variants was accompanied by a change of the leukocyte formula towards a decrease of the lymphocyte percentage and an increase of the granulocyte percentage.

Considering the important role of lymphocytes and neutrophils in many pathological processes, including cancer, some authors consider the diagnostic, prognostic and/or predictive value of the neutrophil-to-lymphocyte ratio. In 
particular, the groups of researchers [19] [20] showed that a decrease of the NLR is associated with a positive prognosis for patients with recurrent BC. Recent studies [21] [22] [23] established an association between the NLR and the sensitivity of tumors of different genesis to chemotherapeutic agents. Most of these studies have analyzed an association between NLR and recurrence-free survival (RFS) in cancer patients, and high NLR value was considered as a marker or predictor of tumor resistance to anticancer therapy [24] [25].

In our study, we have analyzed the existence of a direct relationship between the NLR and the degree of sensitivity (resistance) of the Walker carcinosarcoma to doxorubicin.

According to our data, the development of both carcinosarcoma variants was accompanied by a significant increase of the NLR (compared with the intact control), but there was no correlation between the NLR and the sensitivity of the tumor to doxorubicin. This is indicated by the absence of a significant difference between the NLR values in two groups of tumor-bearing animals.

So, it is clear that the use of the NLR to assess the degree of tumor resistance to chemotherapy is inadequate and requires additional data. In this work, we have analyzed another potential marker of tumor sensitivity to cytotoxic drugs, in particular, the functional state of peripheral blood phagocytes assessed by the ability of these cells to engulf inactivated staphylococci and ROS products.

In the course of the research, we revealed significant differences between some indices of functional activity of neutrophils and monocytes of peripheral blood of rats with a resistant Walker carcinosarcoma variant and animals with a parental variant of the tumor.

Regarding the indices of phagocytic activity, the most informative markers of DOX-resistance were the counts (relative and absolute) of phagocytic monocytes and the intensity of phagocytosis. The levels of these indices of phagocytic activity of peripheral blood monocytes in rats with the DOX-resistant variant was several times higher than the corresponding indices of tumor-free rats and rats with a parental tumor variant. At the same time, changes of the phagocytic activity indices of peripheral blood neutrophils did not correlate with the degree of sensitivity of the tumors to the action of doxorubicin.

The study has revealed an evident relation between the degree of sensitivity/resistance of the tumor to the action of doxorubicin and the ability of both monocytes and neutrophils of peripheral blood to generate ROS. In particular, the level of ROS in neutrophils and monocytes in the blood of rats with the DOX-resistant variant was significantly higher than the corresponding index in rats with a parental tumor type and intact animals.

Interestingly, the growth of the DOX-resistant tumors caused a 4 -fold decrease in the counts (relative and absolute) of ROS generating monocytes. Taking into account the simultaneous increase of the phagocytic monocyte counts, one may conclude on the polarization of these cells against the background of the development of the DOX-resistant tumors. 
It is well known that monocytes are highly plastic and heterogeneous. Due to the expression of various receptors, they monitor and sense environmental changes and readjust their functional phenotype in response to monocyte microenvironment [26]. A significant impact into this microenvironment is made by tumor-derived factors (TDFs) which are produced by tumor cells and tumor-associated stromal network, and are involved in monocyte polarization, their recruitment to tumor and differentiation into distinct TAM subsets [27] [28]. These TDFs include numerous chemokines, cytokines, and various growth factors. A high production level of some TDFs is associated with the resistance of malignant tumors to the action of anticancer drugs. One could mention such TDF as pleiotropic cytokine interleukin-6 (IL-6), one of the major mediators of inflammation. The level of IL-6 expression was shown to be elevated in different cancer cells resistant to anticancer agents including doxorubicin [29] [30] [31].

It should be noted that a significant decrease of the number of ROS producing monocytes was accompanied by a nearly twofold increase of the total ROS level in peripheral blood monocytes of rats with a resistant tumor variant. This indicates a significant intensification of the generation of radicals in each monocyte.

It was reported that ROS plays a critical role in the monocyte/macrophage differentiation to M2 phenotype and the occurrence of tumor-associated macrophages (TAM) [32]. Such a subset of tumor-associated myeloid cells shows an ability to enhance inflammation, to promote resistance against cytotoxic agents (including extracellular pathogens), angiogenesis, tissue remodeling and repair [27]. The emergence of a subset of circulating monocytes with high ROS generation in the rats bearing DOX-resistant tumors correlated with an increase of the TAM counts recorded during histological studies of the tumor tissue.

\section{Conclusion}

We conclude that our study provide an evidence that such indices of the functional activity of circulating phagocytes of the tumor-bearing rats as the quantity and the phagocytosis intensity of monocytes, as well as the intensity of ROS production by monocytes and neutrophils, may reflect the degree of sensitivity of the tumor to doxorubicin, and the increase in these indices could serve as a predictor of the formation of tumor drug resistance. The predictive value of these indices is especially important in the dynamic monitoring of the development of tumor drug resistance during long-term cancer chemotherapy. Considering the standard 2 - 3 week interval between the courses of cancer therapy and the short lifetime of circulating phagocytes, an assessment of the indices of their functional activity before each subsequent course can be considered as a pretreatment assessment. Meanwhile, further studies are needed to determine the spectrum of malignant neoplasms for which the degree of tumor drug resistance correlates with the functional activity of circulating phagocytes.

\section{Conflicts of Interest}

The authors declare no conflicts of interest regarding the publication of this 
paper.

\section{References}

[1] Bray, F., Ferlay, J., Soerjomataram, I., Siegel, R.L., Torre, L.A. and Jemal, A. (2018) Global Cancer Statistics 2018: GLOBOCAN Estimates of Incidence and Mortality Worldwide for 36 Cancers in 185 Countries. CA: A Cancer Journal for Clinicians, 68, 394-424. https://doi.org/10.3322/caac.21492

[2] American Cancer Society (2017) Breast Cancer Facts \& Figures 2017-2018. American Cancer Society, Inc., Atlanta.

https://www.cancer.org/content/dam/cancer-org/research/cancer-facts-and-statistic s/breast-cancer-facts-and-figures/breast-cancer-facts-and-figures-2017-2018.pdf

[3] Gonzalez-Angulo, A.M., Morales-Vasquez, F. and Hortobagyi, G.N. (2000-2013) Overview of Resistance to Systemic Therapy in Patients with Breast Cancer. Madame Curie Bioscience Database. Landes Bioscience, Austin. https://www.ncbi.nlm.nih.gov/books/NBK6306

[4] Li, G., Guo, J., Shen, B.Q., Yadav, D.B., Sliwkowski, M.X., Crocker, L.M., et al. (2018) Mechanisms of Acquired Resistance to Trastuzumab Emtansine in Breast Cancer Cells. Molecular Cancer Therapeutics, 17, 1441-1453. https://doi.org/10.1158/1535-7163.MCT-17-0296

[5] Eisenhauer, E.A., Therasse, P., Bogaerts, J., Schwartz, L.H., Sargent, D., Ford, R., et al. (2009) New Response Evaluation Criteria in Solid Tumours: Revised RECIST Guideline (Version 1.1). European Journal of Cancer, 45, 228-247. https://doi.org/10.1016/j.ejca.2008.10.026

[6] Duffy, M.J. (2012) Tumor Markers in Clinical Practice: A Review Focusing on Common Solid Cancers. Medical Principles and Practice, 22, 4-11. https://doi.org/10.1159/000338393

[7] Araki, K., Ito, Y., Fukada, I., Kobayashi, K., Miyagawa, Y., Imamura, M., Kira, A., et al. (2018) Predictive Impact of Absolute Lymphocyte Counts for Progression-Free Survival in Human Epidermal Growth Factor Receptor 2-Positive Advanced Breast Cancer Treated with Pertuzumab and Trastuzumab plus Eribulin or Nab-Paclitaxel. BMC Cancer, 18, 982. https://doi.org/10.1186/s12885-018-4888-2

[8] Xue, L.B., Liu, Y.H., Zhang, B., Yang, Y.F., Yang, D., Zhang, L.W., et al. (2019) Prognostic Role of High Neutrophil-to-Lymphocyte Ratio in Breast Cancer Patients Receiving Neoadjuvant Chemotherapy: Meta-Analysis. Medicine, 98, e13842. https://doi.org/10.1097/MD.0000000000013842

[9] Dirican, A., Varol, U., Kucukzeybek, Y., Alacacioglu, A., Erten, C., Somali, I., et al. (2014) Treatment of Metastatic Colorectal Cancer with or without Bevacizumab: Can the Neutrophil/Lymphocyte Ratio Predict the Efficiency of Bevacizumab? Asian Pacific Journal of Cancer Prevention, 15, 4781-4786. https://doi.org/10.7314/APJCP.2014.15.12.4781

[10] Todor, I.N., Lukianova, N.Y., Shvets, Y.V., Lozovska, Y.V. and Chekhun, V.F. (2015) Metabolic Changes during Development of Walker-256 Carcinosarcoma Resistance to Doxorubicin. Experimental Oncology, 37, 19-22. https://doi.org/10.31768/2312-8852.2015.37(1):19-22

[11] Pinegin, B.V., Yarilin, A.A., Simonova, A.V., et al. (2001) The Use of Flow Cytometry to Assess the Functional Activity of the Human Immune System. Manual for Laboratory Doctors, Moscow. (In Russian)

[12] Korzhevsky, D.E. (2013) Morphological Diagnosis. Preparation of Material for Histological Examination and Electron Microscopy: A Guide. SPb: SpecLit. (In Rus- 
sian)

[13] Kruger, P., Saffarzadeh, M., Weber, A.N.R., Rieber, N., Radsak, M., von Bernuth, H., et al. (2015) Neutrophils: Between Host Defence, Immune Modulation, and Tissue Injury. PLOS Pathogens, 11, e1004651. https://doi.org/10.1371/journal.ppat.1004651

[14] Wang, X., Qiu, L., Li, Z., Wang, X.Y. and Yi, H. (2018) Understanding the Multifaceted Role of Neutrophils in Cancer and Autoimmune Diseases. Frontiers in Immunology, 9, 2456. https://doi.org/10.3389/fimmu.2018.02456

[15] Finisguerra, V., Di Conza, G., Di Matteo, M., Serneels, J., Costa, S., Thompson, A.A.R., et al. (2015) MET Is Required for the Recruitment of Anti-Tumoural Neutrophils. Nature, 522, 349-353. https://doi.org/10.1038/nature14407

[16] Coffelt, S.B., Kersten, K., Doornebal, C.W., Weiden, J., Vrijland, K., Hau, C.-S., et al. (2015) IL-17-Producing $\gamma \delta$ T Cells and Neutrophils Conspire to Promote Breast Cancer Metastasis. Nature, 522, 345-348. https://doi.org/10.1038/nature14282

[17] Grecian, R., Whyte, M.K.B. and Walmsley, S.R. (2018) The Role of Neutrophils in Cancer. British Medical Bulletin, 128, 5-14. https://doi.org/10.1093/bmb/ldy029

[18] Benarafa, C. (2015) Tumor-Induced Inflammation Alters Neutrophil Phenotype and Disease Progression. Breast Cancer Research, 17, 135. https://doi.org/10.1186/s13058-015-0644-6

[19] Patel, D.A., Xi, J., Luo, J., Hassan, B., Thomas, S., Ma, C.X., et al. (2019) Neutrophil-to-Lymphocyte Ratio as a Predictor of Survival in Patients with Triple-Negative Breast Cancer. Breast Cancer Research and Treatment, 174, 443-452. https://doi.org/10.1007/s10549-018-05106-7

[20] Fujimoto, Y., Ozawa, H., Higuchi, T., Miyagawa, Y., Bun, A., Imamura, M., et al. (2019) Improved Prognosis of Low Baseline Neutrophil-to-Lymphocyteratio Is Significantly Exclusive in Breast Cancer Patients with High Absolute Counts of Lymphocytes. Molecular and Clinical Oncology, 10, 275-284. https://doi.org/10.3892/mco.2018.1783

[21] Imamura, M., Morimoto, T., Egawa, C., Fukui, R., Bun, A., Ozawa, H., et al. (2019) Significance of Baseline Neutrophil-to-Lymphocyte Ratio for Progression-Free Survival of Patients with HER2-Positive Breast Cancer Treated with Trastuzumab Emtansine. Scientific Reports, 9, Article No. 1811. https://doi.org/10.1038/s41598-018-37633-0

[22] Dirican, N., Dirican, A., Anar, C., Atalay, S., Ozturk, O., Bircan, A., et al. (2016) New Inflammatory Prognostic Index, Based on c-Reactive Protein, the Neutrophil to Lymphocyte Ratio and Serum Albumin Is Useful for Predicting Prognosis in Non-Small Cell Lung Cancer Cases. Asian Pacific Journal of Cancer Prevention, 17, 5101-5106.

[23] Botta, C., Barbieri, V., Ciliberto, D., Rossi, A., Rocco, D., Addeo, R., et al. (2013) Systemic Inflammatory Status at Baseline Predicts Bevacizumab Benefit in Advanced Non-Small Cell Lung Cancer Patients. Cancer Biology \& Therapy, 14, 469-475. https://doi.org/10.4161/cbt.24425

[24] Ethier, J.L., Desautels, D., Templeton, A., Shah, P.S. and Amir, E. (2017) Prognostic Role of Neutrophil-to-Lymphocyte Ratio in Breast Cancer: A Systematic Review and Meta-Analysis. Breast Cancer Research, 19, 2. https://doi.org/10.1186/s13058-016-0794-1

[25] Miao, Y., Yan, Q., Li, S., Li, B. and Feng, Y. (2016) Neutrophil to Lymphocyte Ratio and Platelet to Lymphocyte Ratio Are Predictive of Chemotherapeutic Response and Prognosis in Epithelial Ovarian Cancer Patients Treated with Platinum-Based 
Chemotherapy. Cancer Biomarkers, 17, 33-40. https://doi.org/10.3233/CBM-160614

[26] Gordon, S. and Taylor, P.R. (2005) Monocyte and Macrophage Heterogeneity. Nature Reviews Immunology, 5, 953-964. https://doi.org/10.1038/nri1733

[27] Sica, A., Porta, C., Morlacchi, S., Banfi, S., Strauss, L., Rimoldi, M., Totaro, M.G. and Riboldi, E. (2012) Origin and Functions of Tumor-Associated Myeloid Cells (TAMCs). Cancer Microenvironment, 5, 133-149. https://doi.org/10.1007/s12307-011-0091-6

[28] Gyamfi, J., Eom, M., Koo, J.S. and Choi, J. (2018) Multifaceted Roles of Interleukin-6 in Adipocyte-Breast Cancer Cell Interaction. Translational Oncology, 11, 275-285https://doi.org/10.1016/j.tranon.2017.12.009

[29] Mon, M.T., Yodkeeree, S., Punfa, W., Umsumarng, S., Lekwanavijit, S., Siriaunkgul, S., et al. (2017) Relationships of EX-Vivo Drug Resistance Assay and Cytokine Production with Clinicopathological Features in the Primary Cell Culture of Thai Ovarian and Fallopian Tube Cancer Patients. Asian Pacific Journal of Cancer Prevention, 18, 3063-3071.

[30] Li, X.Y., Liao, X.F., Wang, H.B. and Zhang, J. (2018) Doxorubicin Resistance Induces IL6 Activation in the Colon Cancer Cell Line LS180. Oncology Letters, 16, 5923-5929. https://doi.org/10.3892/ol.2018.9360

[31] Chen, Y., Liu, J., Lv, P., Gao, J., Wang, M. and Wang, Y. (2018) IL-6 Is Involved in Malignancy and Doxorubicin Sensitivity of Renal Carcinoma Cells. Cell Adhesion \& Migration, 12, 28-36. https://doi.org/10.1080/19336918.2017.1307482

[32] Zhang, Y., Choksi, S., Chen, K., Pobezinskaya, Y., Linnoila, I. and Liu, Z.G. (2013) ROS Play a Critical Role in the Differentiation of Alternatively Activated Macrophages and the Occurrence of Tumor-Associated Macrophages. Cell Research, 23, 898-914. https://doi.org/10.1038/cr.2013.75 